\title{
ON THE GEVREY WELL-POSEDNESS FOR SECOND ORDER STRICTLY HYPERBOLIC CAUCHY PROBLEMS UNDER THE INFLUENCE OF THE REGULARITY OF THE COEFFICIENTS
}

\author{
MASSIMO CICOGNANI and FUMIHIKO HIROSAWA
}

\begin{abstract}
We consider the loss of regularity of the solution to the backward Cauchy problem for a second order strictly hyperbolic equation on the time interval $[0, T]$ with time depending coefficients which have a singularity only at the end point $t=0$. The main purpose of this paper is to show that the loss of regularity of the solution on the Gevrey scale can be described by the order of differentiability of the coefficients on $(0, T]$, the order of singularities of each derivatives as $t \rightarrow 0$ and a stabilization condition of the amplitude of oscillations described by an integral on $(0, T)$. Moreover, we prove the optimality of the conditions for $C^{\infty}$ coefficients on $(0, T]$ by constructing a counterexample.
\end{abstract}

\section{Introduction}

Let us consider the following backward Cauchy problem for a second order strictly hyperbolic equation:

$$
\begin{cases}\left(\partial_{t}^{2}-a(t)^{2} \Delta\right) u(t, x)=0, & (t, x) \in[0, T) \times \mathrm{R}^{n}, \\ u(T, x)=u_{0}(x), & \left(\partial_{t} u\right)(T, x)=u_{1}(x), x \in \mathrm{R}^{n},\end{cases}
$$

where $T$ is a positive small constant and $a(t)$ satisfies

$$
a_{0} \leq a(t) \leq a_{1}
$$

for some positive constants $a_{0}$ and $a_{1}$.

We are interested in the loss of regularity of the solution due to singularities of the coefficients. In particular, we focus on the $L^{2}$ and Gevrey well-posedness of (1.1) in the case that the coefficients are singular only at $t=0$; thus we assume that $a(t) \in C^{\infty}((0, T])$. Here we adopt the following definition of the

Received September 19, 2006. 
(inductive) Gevrey class $\gamma^{(s)}$ with $s>1$ taking the Paley-Wiener theorem into account: $\gamma^{(s)}=\bigcup_{\rho>0} \gamma_{\rho}^{(s)}$, where

$$
\gamma_{\rho}^{(s)}=\left\{f(x) \in C_{0}^{\infty}\left(\mathrm{R}^{n}\right) ; \sup _{\xi \in \mathrm{R}^{n}}\left\{\exp \left(-\rho\langle\xi\rangle^{\frac{1}{s}}\right)|\hat{f}(\xi)|\right\}<\infty\right\}
$$

and $\hat{f}(\xi)$ denotes the Fourier transform of $f(x)$ with respect to the space variable $x$.

One of the earliest results for such a problem is the following, contained in [3]:

TheOREM 1.1 ([3]). Let $\sigma \geq 1$ be a real number. Assume that a $(t)$ satisfies

$$
\left|a^{\prime}(t)\right| \leq C t^{-\sigma}
$$

for a positive constant $C$. If $\sigma>1$, then (1.1) is $\gamma^{(s)}$ well-posed for $s<$ $\sigma /(\sigma-1)$. If $\sigma=1$, then (1.1) is $C^{\infty}$ well-posed. On the other hand, there exists a(t) satisfying $1 / 2 \leq a(t) \leq 3 / 2$ and (1.2) such that (1.1) is not $\gamma^{(s)}$ well-posed for any $s>\sigma /(\sigma-1)$.

REMARK 1.1. If $\sigma<1$, then $a^{\prime}(t) \in L^{1}((0, T))$, which immediately gives the $L^{2}$ well-posedness of (1.1). For $\sigma=1$, the $C^{\infty}$ well-posedness in Theorem 1.1 is proved with a loss of derivatives.

The equation of (1.1) describes forced oscillations, so it is natural to discuss the relations between the stability of the solution and the speed of oscillation $\left|a^{\prime}(t)\right|$ of the coefficient. The regularity of the solution is conserved on $[0, T]$ if $\left|a^{\prime}(t)\right|$ is bounded, therefore one comes to consider unbounded derivatives as in (1.2). Theorem 1.1 shows that the related classification of well-posedness is natural in the scale of Gevrey spaces. The conclusions of Theorem 1.1 are optimal, however, an additional condition on the second order derivative improves the result in the case $\sigma=1$ as follows:

Theorem 1.2 ([5] (cf. [7])). Assume that a $(t)$ satisfies

$$
\left|a^{(k)}(t)\right| \leq C_{k} t^{-k} \quad \text { for } k=1,2,
$$

where $a^{(k)}(t)=\frac{d^{k}}{d t^{k}} a(t)$. Then (1.1) is $L^{2}$ well-posed.

Remark 1.2. We shall denote universal positive constants by $C$ and $C_{k}$ $(k=0,1, \ldots)$.

Remark 1.3. The $L^{2}$ well-posedness of (1.1) with the coefficient $a(t)=$ $2+\cos (\log (1 / t))$ follows from Theorem 1.2 but cannot be obtained by Theorem 1.1. 
The additional condition on the second order derivative in Theorem 1.2 is required for a precise representation of WKB solutions of (1.1). If $a(t)=a$ is a constant coefficient, then (1.1) can be reduced to a completely diagonal first order system, otherwise the equivalent system operator contains antidiagonal elements where the factor $a^{\prime}(t)$ appears. In a certain sense, the results of Theorem 1.1 are proved by means of a diagonalization procedure in the WKB analysis which takes only the $C^{1}$ regularity of the coefficient $a(t)$ into account, thus we call this method $C^{1}$ property. On the other hand, the conclusion of Theorem 1.2 is proved by introducing one more step of diagonalization where the $C^{2}$ regularity of the coefficient $a(t)$ is used, so we call this method $C^{2}$ property. In the $C^{2}$ theory, we have a more precise representations of WKB solutions which gives the $L^{2}$ well-posedness under the assumptions of (1.3). In other words, the $C^{2}$ property provides a reduced system whose anti-diagonal components are negligible errors for the $L^{2}$ well-posedness.

The well-posedness of (1.1) has been investigated also in relation to the modulus of continuity of the coefficients. If $a(t)$ is Lipschitz continuous on $[0, T]$, then (1.1) is obviously $L^{2}$ well-posed. On the other hand, one cannot expect in general the $L^{2}$ well-posedness if $a(t)$ is not a Lipschitz continuous function. This means that some singular behavior of the coefficient with respect to the Lipschitz continuity brings loss of regularity of the solution. We are referring to the following well known result:

THEOREM 1.3 ([1]). Let $\alpha$ be a real number satisfying $0<\alpha<1$. Assume that a(t) satisfies

$$
\sup _{\tau \in[0, T]}\left\{\int_{0}^{t}|a(\tau+s)-a(\tau)| d s\right\} \leq C t^{\alpha+1} .
$$

Then (1.1) is $\gamma^{(s)}$ well-posed for $s<1 /(1-\alpha)$. Moreover, there exists $a(t)$ satisfying $1 / 2 \leq a(t) \leq 3 / 2$ and (1.4) such that (1.1) is not $\gamma^{(s)}$ well-posed for any $s>1 /(1-\alpha)$.

REMARK 1.4. In [1] the stronger condition $a(t) \in C^{\alpha}([0, T])$ is assumed, where $C^{\alpha}([0, T])$ denote the class of Hölder continuous functions on $[0, T]$ of exponent $\alpha$. However, (1.4) is the condition used in the proof.

It is also considered in [3] the mixed case of the conditions (1.2) and (1.4) as follows:

TheOREM 1.4 ([3]). Let $\alpha$ and $\sigma$ be real numbers satisfying $0<\alpha<1$ and $\sigma>1$. If $a(t)$ satisfies (1.2) and (1.4), then (1.1) is $\gamma^{(s)}$ well-posed for $s<\sigma /((1-\alpha)(\sigma-1))$.

We observe from Theorem 1.1 to 1.4 the following properties: 
(i) The order of loss of regularity of the solution is described by the oscillation speed of $a(t)$ near $t=0$, that is by the behavior of $\left|a^{\prime}(t)\right|$ (from Theorem 1.1).

(ii) If we assume the further condition (1.4), which describes some stabilization of the amplitude of $a(t)$, besides (1.2), then we have less loss of regularity (from Theorem 1.4).

(iii) Further assumptions on higher order derivatives of the coefficient can provide some improvements (from Theorem 1.2).

We introduce a further result, which supports the observation (iii).

THEOREM 1.5 ([2]). Let $m$ be a positive integer, $\alpha$ and $\sigma$ be real numbers satisfying $0<\alpha<1, \sigma>(1-\alpha / m) /(1-\alpha)$. If $a(t) \in C^{m}((0, T]) \cap$ $C^{\alpha}([0, T])$ satisfies

$$
\left|a^{(k)}(t)\right| \leq C_{k} t^{-k \sigma} \quad \text { for } \quad k=1, \ldots, m,
$$

then (1.1) is $\gamma^{(s)}$ well-posed for

$$
s<\tilde{s}_{m}:=\frac{\sigma}{\sigma(1-\alpha)+\frac{\alpha}{m}-1} .
$$

Remark 1.5. In the case $m=1$, Theorem 1.5 reduces to Theorem 1.4. In general we have $\tilde{s}_{m}>\tilde{s}_{1}$ for $m \geq 2$, which implies that the additional assumptions on the higher order derivatives ensure the well-posedness in a wider class under the same condition on the oscillation speed $\left|a^{\prime}(t)\right|$ near $t=0$.

Remark 1.6. The original result in [2] is proved under the sole assumption (1.5) with $k=m$ on the highest order derivative $a^{(m)}(t)$.

The main purpose of this paper is to obtain results in the direction of observation (iii) as in Theorem 1.5, but considering such a problem from an essentially different point of view. The details of the difference will be discussed by the aid of Example 2.1. Our main result will be introduced in the next section, here we state a corollary of it, cf. Theorem 1.5.

Corollary 1.1 (from Theorem 2.1). Let $m$ be a positive integer, $\mu$ and $\sigma$ be real numbers satisfying $\sigma>\mu+1-\mu / m$. If a (t) satisfies (1.5) and

$$
\int_{0}^{t}|a(s)-a(0)| d s \leq C t^{\mu+1}
$$


then (1.1) is $\gamma^{(s)}$ well-posed for

$$
s<s_{m}:=\frac{\sigma+\frac{\mu}{m}}{\sigma+\frac{\mu}{m}-\mu-1} .
$$

In particular, if $m \geq 2$ and (1.5) holds for $\sigma=\mu+1-\mu / m$, then (1.1) is $L^{2}$ well-posed.

The proof of the corollary will be given in the appendix.

Corollary 1.1 generalizes Theorem 1.1 and Theorem 1.2. In fact, if $m=1$, $\sigma>1$ and $\mu=0$, then $s_{1}=\sigma /(\sigma-1)$. If $m=2, \sigma=1$ and $\mu=0$, then the conclusion from Corollary 1.1 corresponds to Theorem 1.2. Moreover, $s_{m}$ is monotone increasing with respect to $m$ since $\mu>0$. Obviously, a $C^{\alpha}$ function, $\alpha<1$, satisfies (1.6) with $\mu=\alpha$ and we have $s_{m}>\tilde{s}_{m}$ if we put $\alpha=\mu$ in the definitions of these indices of well-posedness. So, Corollary 1.1 extends also Theorem 1.5.

\section{The main theorem and a typical example}

Let us say that $\Theta=\Theta(t) \in C^{0}([0, T])$ is a reference function if $\Theta(t)$ is monotone increasing, $\Theta(0)=0, \Theta(t)>0$ for any $t>0, \sup _{t \in(0, T)}\{\Theta(t) / t\}<\infty$ and $\sup _{t \in(0, T)}\{\Theta(2 t) / \Theta(t)\}<\infty$. We introduce a class of functions, which describes a stabilization of the amplitude by the reference function $\Theta$ as follows:

$$
L((0, T) ; \Theta)=\left\{f(t) \in L^{\infty}((0, T)) ; \int_{0}^{t}|f(s)-f(0)| d s \leq C \Theta(t)\right\} .
$$

In particular, if $\Theta(t)=t^{\alpha}$ for $\alpha>1$, we denote $L((0, T) ; \Theta)$ by $L^{(\alpha)}((0, T))$. Actually, the reference function $\Theta(t)$ should satisfy the following property:

$$
\lim _{t \rightarrow 0} \frac{\Theta(t)}{t}=0
$$

otherwise, the condition $a(t) \in L((0, T) ; \Theta)$ is true for any bounded function $a(t)$.

After having introduced the class $L((0, T) ; \Theta)$, we can now state our main theorem:

THEOREM 2.1. Let $m$ be a positive integer, $\beta$ be a real number satisfying $\beta \geq 1$ and $\Theta$ be a reference function satisfying (2.1). Assume that $a(t) \in$ $C^{m}((0, T]) \cap L((0, T) ; \Theta)$ satisfies

$$
\left|a^{(k)}(t)\right| \leq C_{k}\left(\frac{1}{\Theta(t)^{\beta}}\left(\frac{\Theta(t)}{t}\right)^{\frac{1}{m}}\right)^{k} \quad \text { for } \quad k=1, \ldots, m .
$$


If $\beta>1$, then (1.1) is $\gamma^{(s)}$ well-posed for $s<\beta /(\beta-1)$. If $\beta=1$ and $m \geq 2$, then (1.1) is $L^{2}$ well-posed.

Theorem 2.1 and the corresponding Corollary 1.1 will be proved using the $C^{m}$ regularity of $a(t)$ on $(0, T]$ and $m$ steps of diagonalization; we call this method $C^{m}$ property. Incidentally, we can naturally introduce the following theorem and corresponding corollary as consequences of the $C^{\infty}$ property.

Theorem 2.2. Let $\beta$ be a positive real number. Assume that $a(t) \in$ $C^{\infty}((0, T]) \cap L((0, T) ; \Theta)$ satisfies

$$
\left|a^{(k)}(t)\right| \leq C_{k} \Theta(t)^{-k \beta}
$$

for any $k \in \mathrm{N}$. If $\beta>1$, then (1.1) is $\gamma^{(s)}$ well-posed for $s<\beta /(\beta-1)$, and if $\beta<1$, then (1.1) is $L^{2}$ well-posed.

Corollary 2.1. Let $\mu$ and $\sigma$ be positive real numbers. Assume that $a(t) \in$ $C^{\infty}((0, T]) \cap L^{(\mu+1)}((0, T))$ satisfies (1.5) for any $m$. If $\sigma>\mu+1$, then (1.1) is $\gamma^{(s)}$ well-posed for $s<\sigma /(\sigma-\mu-1)$, and if $\sigma<\mu+1$, then (1.1) is $L^{2}$ well-posed.

Moreover, the optimality of the condition (2.3) for the conclusion of Theorem 2.2 is ensured by the following theorem:

THeOREM 2.3. For any $\beta>1$ and any reference function $\Theta(t)$ satisfying (2.1), there exists a $(t) \in C^{\infty}((0, T]) \cap L((0, T) ; \Theta)$ satisfying $1 / 2 \leq a(t) \leq$ $3 / 2$ and (2.3) such that (1.1) is not well-posed in $\gamma^{(s)}$ for any $s>\beta /(\beta-1)$.

We introduce now a typical example of $a(t)$ to which Theorem 2.1 and Corollary 1.1 apply, but that cannot be handled by any results of well-posedness from previous papers quoted in the introduction.

ExAmple 2.1. Let $\chi(\tau) \in C_{0}^{\infty}(\mathrm{R})$ satisfying the following properties:

$$
\begin{aligned}
& \operatorname{supp} \chi(\tau) \subset[-1,1], \quad 0 \leq \chi(\tau) \leq 1, \\
& \chi(\tau)=\chi(-\tau) \quad \text { and } \quad \int_{-1}^{1} \chi(\tau) d \tau=1 .
\end{aligned}
$$

For non-negative real numbers $p, q$ and $r$ satisfying

$$
r<1<q-p
$$

and a large integer $N_{0}$ satisfying $N_{0}^{-p}+N_{0}^{-q} \leq T$, we define $a(t)$ by

$$
a(t)=a_{0}+\sum_{j=N_{0}}^{\infty} j^{-r} \chi\left(j^{q}\left(t-j^{-p}\right)\right) .
$$


Here we note that supp $\chi\left(j^{q}\left(t-j^{-p}\right)\right) \cap \operatorname{supp} \chi\left(\ell^{q}\left(t-\ell^{-p}\right)\right)=\emptyset$ for $j \neq \ell$ and $j, \ell \geq N_{0}$ by (2.5). Let us fix $t \in(0, T)$, and set the positive integer $N$ satisfying $(N+1)^{-p} \leq t<N^{-p}$. Then we have

$$
\int_{0}^{t}|a(\tau)-a(0)| d \tau \simeq \sum_{j=N}^{\infty} j^{-q-r} \simeq N^{-q-r+1},
$$

and it follows that $a(t) \in C^{\infty}((0, T]) \cap L^{\left(\frac{q+r-1}{p}\right)}((0, T))$. Moreover, for any fixed positive integer $m$ we have

$$
\left|a^{(k)}(t)\right| \leq C_{k} t^{-k\left(\frac{m q-r}{m p}\right)} .
$$

Here we note that $a(t) \in C^{r / q}([0, T])$ and (1.4) is not valid for any $\alpha>r / q$. Hence we have, from Theorem 1.5 with $\alpha=r / q$ and $\sigma=(m q-r) /(m p)$, that (1.1) is $\gamma^{(s)}$ well-posed for

$$
s<\tilde{s}_{m}=\frac{q}{q-r-p} .
$$

On the other hand, Corollary 1.1 with $\mu=(q+r-1) / p-1$ and $\sigma=$ $(m q-r) /(m p)$ gives the $\gamma^{(s)}$ well-posedness of $(1.1)$ for

$$
s<s_{m}=\frac{q(m+1)-p-1}{q-p-1+m(1-r)} .
$$

Note that $s_{m}>\tilde{s}_{m}$ since $(q-p-1)(m q-p-r)>0$, which is ensured by (2.5). (For calculations in more details, we refer to Appendix.)

The function $a(t)$ of Example 2.1 is a typical one which we want to deal with in this paper. Indeed, we observe the following properties:

- Decaying amplitude and sparse oscillation of a (t) contribute to the stability of the solution: $s_{m}$ (and also $\tilde{s}_{m}$ ) is monotone increasing with respect to $r$ and $p$, which describe faster decay of the amplitude and longer frequency of the oscillations respectively.

- If we apply Theorem 1.5 to a $(t)$, we have no benefit from $C^{m}$ regularity of $a(t)$ for $m \geq 2$ : The Gevrey index $\tilde{s}_{m}$ in (2.6) is independent of $m$; thus the $C^{m}$ regularity of $a(t)$ on $(0, T]$ with $m \geq 2$ gives no contribution.

- Higher oscillation speed may have a good influence: Suppose that $r=0$ for simplicity. Then the oscillation speed of $a(t)$ is higher the larger is $q$. On the other hand, $s_{m}$ is strictly increasing with respect to $q$ if $m>p$. Taking here $m=\infty$, we have that (1.1) is $\gamma^{(s)}$ well-posed for $s<q$, so we have a larger class of well-posedness for a higher speed of oscillation. 
Our main theorems with Example 2.1 develop the essential problem on the strictly hyperbolic equation of (1.1) "which properties of the coefficients lead to small perturbations of the wave equation with constant propagation speed?" Indeed, most of the previous papers dealt with such a problem by controlling the oscillation speed in some form. Following another point of view, our theorems propose that smoothness of the coefficient and its integral in the definition of $L((0, T) ; \Theta)$ should be essential.

\section{Proof of Theorem 2.1}

Let us suppose that the initial data are functions with compact support. The proof of Theorem 2.1 will be carried out by the usual microenergy estimates in $[0, T] \times \mathrm{R}_{\xi}^{n}$ and Paley-Wiener theorem, where $\xi$ is the dual variable of the space variable $x$ in the Fourier transform. The proof consists of the following three steps.

In the first step we consider a regularization of $a(t)$ near $t=0$ and its estimates. Indeed, the regularity of the solution is not influenced by the oscillating behavior of $a(t)$ but by the stabilization of the amplitude near $t=0$. Hence the regularization has to take the property $a(t) \in L((0, T) ; \Theta)$ into account. The second step is a diagonalization procedure in the high frequency part of the phase space where the hyperbolicity of the equation and the $C^{1}$ regularity of $a(t)$ are the main tools. Actually, this step is a typical microenergy estimate. The third step is the main part of the proof. In this step we introduce a refined diagonalization procedure in the high frequency part, which is performed by means of the $C^{m}$ regularity of $a(t)$ under the assumptions (2.2). Indeed, we can derive some good estimates in high frequency part from this procedure; this step is the core of what we call $C^{m}$ property.

\subsection{Regularization of the coefficient}

Let us extend $a(t)$ as an even function for $t<0$. For a positive real number $\varepsilon$, which will depend on $\xi$, we define $\tilde{a}(t)=\tilde{a}(t ; \varepsilon)$ by

$$
\tilde{a}(t ; \varepsilon)=a(t)\left(1-\chi\left(\varepsilon^{-1} t\right)\right)+a * \chi_{\varepsilon}(t) \chi\left(\varepsilon^{-1} t\right),
$$

where $a * \chi_{\varepsilon}(t)=\varepsilon^{-1} \int a(s) \chi\left(\varepsilon^{-1}(t-s)\right) d s$ and $\chi(\tau) \in C_{0}^{\infty}(\mathrm{R})$ satisfies (2.4), $\chi(\tau) \equiv 1$ on $[-1 / 2,1 / 2]$ and $\int \chi^{(k)}(\tau) d \tau=0$ for any positive integer $k$. Then we have the following lemmas:

Lemma 3.1. If $a(t) \in L((0, T) ; \Theta)$, then the following estimate holds:

$$
\int_{0}^{T}|\tilde{a}(t ; \varepsilon)-a(t)| d t \leq C \Theta(\varepsilon) .
$$


Proof. Noting the estimates

$$
\begin{aligned}
\left|a * \chi_{\varepsilon}(t)-a(0)\right| & \leq \varepsilon^{-1} \int_{-\varepsilon}^{\varepsilon}|a(t-\tau)-a(0)| \chi\left(\varepsilon^{-1} \tau\right) d \tau \\
& \leq C \varepsilon^{-1} \Theta(\varepsilon)
\end{aligned}
$$

for $t \in[0, \varepsilon]$, we have

$$
\begin{aligned}
\int_{0}^{T}|\tilde{a}(t ; \varepsilon)-a(t)| d t & \leq \int_{0}^{\varepsilon}\left|a * \chi_{\varepsilon}(t)-a(0)\right| d t+\int_{0}^{\varepsilon}|a(t)-a(0)| d t \\
& \leq C \Theta(\varepsilon) .
\end{aligned}
$$

Lemma 3.2. If $a(t) \in L((0, T) ; \Theta) \cap C^{m}((0, T])$ satisfies $(2.2)$, then the following estimates hold:

$$
\begin{aligned}
& \left|\frac{d^{k}}{d t^{k}} \tilde{a}(t ; \varepsilon)\right| \\
& \quad \leq C_{k}\left(\left(\frac{1}{\Theta(t)^{\beta}}\left(\frac{\Theta(t)}{t}\right)^{\frac{1}{m}}\right)^{k} \phi(t ;[\varepsilon / 2, T])+\varepsilon^{-k-1} \Theta(\varepsilon) \phi(t ;[0, \varepsilon])\right)
\end{aligned}
$$

for any $k=1, \ldots, m$, where $\phi(t ; I)$ denotes the characteristic function on $I$.

Proof. For $t \in[0, \varepsilon]$ and $1 \leq j \leq k$, we have

$$
\begin{aligned}
\left|\frac{d^{j}}{d t^{j}} a * \chi_{\varepsilon}(t)\right| & =\left|\frac{d^{j}}{d t^{j}} a * \chi_{\varepsilon}(t)-\varepsilon^{-j} a(0) \int \chi^{(j)}(\tau) d \tau\right| \\
& =\varepsilon^{-j}\left|\int_{-1}^{1} \chi^{(j)}(\tau)(a(t-\varepsilon \tau)-a(0)) d \tau\right| \\
& \leq \varepsilon^{-j-1} \int_{-\varepsilon}^{\varepsilon}|a(t-s)-a(0)| d s \\
& \leq \varepsilon^{-j-1} \int_{-2 \varepsilon}^{2 \varepsilon}|a(\tau)-a(0)| d \tau \\
& \leq C \varepsilon^{-j-1} \Theta(\varepsilon) .
\end{aligned}
$$


Therefore, noting the estimates

$$
\begin{aligned}
\varepsilon^{-k+j}\left(\frac{1}{\Theta(t)^{\beta}}\left(\frac{\Theta(t)}{t}\right)^{\frac{1}{m}}\right)^{j} & \leq\left(\frac{1}{\Theta(t)^{\beta}}\left(\frac{\Theta(t)}{t}\right)^{\frac{1}{m}}\right)^{k}\left(\frac{t}{\Theta(t)^{\beta}}\left(\frac{\Theta(t)}{t}\right)^{\frac{1}{m}}\right)^{j-k} \\
& \leq\left(\frac{1}{\Theta(t)^{\beta}}\left(\frac{\Theta(t)}{t}\right)^{\frac{1}{m}}\right)^{k} \Theta(t)^{(\beta-1)(k-j)} \\
& \leq\left(\frac{1}{\Theta(t)^{\beta}}\left(\frac{\Theta(t)}{t}\right)^{\frac{1}{m}}\right)^{k}
\end{aligned}
$$

for $t \in[0, \varepsilon]$ and $\tilde{a}(t ; \varepsilon)=a(t)$ for $t \in[\varepsilon, T]$, we obtain

$$
\begin{aligned}
& \left|\frac{d^{k}}{d t^{k}} \tilde{a}(t ; \varepsilon)\right| \\
& \leq\left|a^{(k)}(t)\right| \phi(t ;[\varepsilon / 2, T])+\left|\frac{d^{k}}{d t^{k}} a * \chi_{\varepsilon}(t)\right| \phi(t ;[0, \varepsilon]) \\
& \quad+\sum_{j=0}^{k-1}\left(\begin{array}{c}
k \\
j
\end{array}\right) \varepsilon^{-k+j}\left(\left|a^{(j)}(t)\right|+\left|\frac{d^{j}}{d t^{j}} a * \chi_{\varepsilon}(t)\right|\right) \phi(t ;[\varepsilon / 2, \varepsilon]) \\
& \leq C_{k}\left(\left(\frac{1}{\Theta(t)^{\beta}}\left(\frac{\Theta(t)}{t}\right)^{\frac{1}{m}}\right)^{k} \phi(t ;[\varepsilon / 2, T])+\varepsilon^{-k-1} \Theta(\varepsilon) \phi(t ;[0, \varepsilon])\right)
\end{aligned}
$$

for any $t \in[0, T]$.

\subsection{First step of diagonalization procedure $-C^{1}$ property}

By partial Fourier transform with respect to $x,(1.1)$ is rewritten as follows:

$$
\begin{cases}\left(D_{t}^{2}-a(t)^{2}|\xi|^{2}\right) v(t, \xi)=0, & (t, \xi) \in[0, T) \times \mathrm{R}^{n} \\ v(T, \xi)=\hat{u}_{0}(\xi), \quad\left(\partial_{t} v\right)(T, \xi)=\hat{u}_{1}(\xi), & \xi \in \mathrm{R}^{n}\end{cases}
$$

where $v(t, \xi)=\hat{u}(t, \xi)$ and $D=-i \partial$.

Let us arbitrarily fix $\xi \in \mathbf{R}^{n}$ for $|\xi| \gg 1$. Indeed, we are only interested in the behavior of large $|\xi|$, hence such a restriction is not essential. For a positive parameter $\varepsilon=\varepsilon(\xi)$ we define $V=V(t, \xi)$ by

$$
V=V(t, \xi)=\left(\begin{array}{c}
\tilde{a}(t, \xi)|\xi| v(t, \xi) \\
D_{t} v(t, \xi)
\end{array}\right)
$$

where $\tilde{a}(t, \xi)=\tilde{a}(t ; \varepsilon(\xi))$. Then the equation of (3.1) is represented as the following system:

$$
\left(D_{t}-A+B+R\right) V=0,
$$


where

$$
\begin{gathered}
A=A(t, \xi)=\left(\begin{array}{cc}
0 & \tilde{a}(t, \xi)|\xi| \\
\tilde{a}(t, \xi)|\xi| & 0
\end{array}\right), \\
B=B(t, \xi)=-\frac{D_{t} \tilde{a}(t, \xi)}{\tilde{a}(t, \xi)}\left(\begin{array}{ll}
1 & 0 \\
0 & 0
\end{array}\right)
\end{gathered}
$$

and

$$
R=R(t, \xi)=\frac{\left(\tilde{a}(t, \xi)^{2}-a(t)^{2}\right)|\xi|}{\tilde{a}(t, \xi)}\left(\begin{array}{ll}
0 & 0 \\
1 & 0
\end{array}\right)
$$

We define $M_{0}$ and $V_{1}=V_{1}(t, \xi)$ by

$$
M_{0}=\left(\begin{array}{rr}
1 & -1 \\
1 & 1
\end{array}\right) \quad \text { and } \quad V_{1}(t, \xi)=M_{0}^{-1} V(t, \xi)
$$

Then we have

$$
M_{0}^{-1}\left(D_{t}-A+B+R\right) M_{0}=D_{t}-\Phi_{1}+B_{1}+R_{1},
$$

where

$$
\begin{aligned}
& \Phi_{1}=\Phi_{1}(t, \xi)=\left(\begin{array}{cc}
\tau_{1-} & 0 \\
0 & \tau_{1+}
\end{array}\right), \tau_{1 \pm}=\tau_{1 \pm}(t, \xi)=\mp \tilde{a}(t, \xi)|\xi|+\frac{D_{t} \tilde{a}(t, \xi)}{2 \tilde{a}(t, \xi)} \\
& B_{1}=B_{1}(t, \xi)=i\left(\begin{array}{cc}
0 & \overline{\beta_{1}(t)} \\
\beta_{1}(t) & 0
\end{array}\right), \quad \beta_{1}=\beta_{1}(t)=-\frac{i D_{t} \tilde{a}(t, \xi)}{2 \tilde{a}(t, \xi)}\left(=\overline{\beta_{1}}\right)
\end{aligned}
$$

and $R_{1}=M_{0}^{-1} R M_{0}$, and it follows that $V_{1}$ is a solution of the equation

$$
\left(D_{t}-\Phi_{1}+B_{1}+R_{1}\right) V_{1}=0 \text {. }
$$

We only used $C^{1}$ regularity of $a(t)$ on $(0, T]$ at this moment, hence this step can be called $C^{1}$-property. Indeed, Theorem 1.1 is immediately proved from the reduced equation (3.2).

\subsection{Refined diagonalization procedure $-C^{m}$ property}

We shall carry out further steps of diagonalization procedure making use of the $C^{m}$ regularity of $a(t)$. The crucial points of this procedure are the regularity of the coefficient and the symmetricity of the characteristic roots. (Originally, this method was introduced in [6].)

Let us assume the following hypotheses: 
(i) $B_{j}=B_{j}(t, \xi)$ is represented by

$$
B_{j}(t, \xi)=i\left(\begin{array}{cc}
0 & \overline{\beta_{j}(t, \xi)} \\
\beta_{j}(t, \xi) & 0
\end{array}\right)
$$

(ii) $\Phi_{j}=\Phi_{j}(t, \xi)$ is represented by

$$
\Phi_{j}(t, \xi)=\left(\begin{array}{cc}
\tau_{j-}(t, \xi) & 0 \\
0 & \tau_{j+}(t, \xi)
\end{array}\right)
$$

where $\phi_{j}=\phi_{j}(t, \xi):=\tau_{j+}(t, \xi)-\tau_{j-}(t, \xi)$ is real-valued.

(iii) The matrix $M_{j}=M_{j}(t, \xi)$ :

$$
M_{j}(t, \xi)=I+\frac{i}{\phi_{j}(t, \xi)}\left(\begin{array}{cc}
0 & -\overline{\beta_{j}(t, \xi)} \\
\beta_{j}(t, \xi) & 0
\end{array}\right)
$$

is invertible, that is, $\left|\beta_{j}(t, \xi)\right|^{2} / \phi_{j}(t, \xi)^{2}=: d_{j}(t, \xi)<1$ uniformly with respect to $t$ and $\xi$, where $I$ denotes the identity matrix.

Then the following equalities are straightforward:

$$
\begin{aligned}
M_{j}^{-1}\left(\Phi_{j}-B_{j}\right) M_{j} & \\
& =\frac{1}{1-d_{j}}\left(\left(\begin{array}{cc}
\tau_{j-}-d_{j} \tau_{j+}+\frac{2\left|\beta_{j}\right|^{2}}{\phi_{j}} & 0 \\
0 & \tau_{j+}-d_{j} \tau_{j-}-\frac{2\left|\beta_{j}\right|^{2}}{\phi_{j}}
\end{array}\right)-d_{j} B_{j}\right)
\end{aligned}
$$

and

$$
\begin{aligned}
& M_{j}^{-1}\left(D_{t} M_{j}\right) \\
& =\frac{1}{1-d_{j}}\left(\left(\begin{array}{cc}
0 & -\partial_{t}\left(\frac{\overline{\beta_{j}}}{\phi_{j}}\right) \\
\partial_{t}\left(\frac{\beta_{j}}{\phi_{j}}\right) & 0
\end{array}\right)+\left(\begin{array}{cc}
i \frac{\overline{\beta_{j}}}{\phi_{j}} \partial_{t}\left(\frac{\beta_{j}}{\phi_{j}}\right) & 0 \\
0 & i \frac{\beta_{j}}{\phi_{j}} \partial_{t}\left(\frac{\overline{\beta_{j}}}{\phi_{j}}\right)
\end{array}\right)\right) .
\end{aligned}
$$

Consequently, we obtain

$$
M_{j}^{-1}\left(D_{t}-\Phi_{j}+B_{j}+R_{j}\right) M_{j}=D_{t}-\Phi_{j+1}+B_{j+1}+R_{j+1},
$$

where

$$
\Phi_{j+1}=\left(\begin{array}{cc}
\tau_{(j+1)-} & 0 \\
0 & \tau_{(j+1)+}
\end{array}\right), \quad B_{j+1}=\left(\begin{array}{cc}
0 & \left(B_{j+1}\right)_{12} \\
\left(B_{j+1}\right)_{21} & 0
\end{array}\right),
$$




$$
\begin{aligned}
\tau_{(j+1)+} & =\frac{1}{1-d_{j}}\left(\tau_{j+}-d_{j} \tau_{j-}-\frac{2\left|\beta_{j}\right|^{2}}{\phi_{j}}+i \frac{\beta_{j}}{\phi_{j}} \partial_{t}\left(\overline{\frac{\beta_{j}}{\phi_{j}}}\right)\right), \\
\tau_{(j+1)-} & =\frac{1}{1-d_{j}}\left(\tau_{j-}-d_{j} \tau_{j+}+\frac{2\left|\beta_{j}\right|^{2}}{\phi_{j}}+i \frac{\overline{\beta_{j}}}{\phi_{j}} \partial_{t}\left(\frac{\beta_{j}}{\phi_{j}}\right)\right), \\
\left(B_{j+1}\right)_{12} & =\frac{i}{1-d_{j}}\left(d_{j} \overline{\beta_{j}}+i \partial_{t}\left(\frac{\overline{\beta_{j}}}{\phi_{j}}\right)\right), \\
\left(B_{j+1}\right)_{21} & =\frac{i}{1-d_{j}}\left(d_{j} \beta_{j}-i \partial_{t}\left(\frac{\beta_{j}}{\phi_{j}}\right)\right)
\end{aligned}
$$

and $R_{j+1}=M_{j}^{-1} R_{j} M_{j}$. We supposed that $\phi_{j}$ is real-valued, hence $\tau_{(j+1) \pm}$, $\left(B_{j+1}\right)_{12}$ and $\left(B_{j+1}\right)_{21}$ are represented as follows:

$$
\begin{aligned}
\tau_{(j+1) \pm} & =\tau_{j \pm} \mp \frac{1}{1-d_{j}}\left(d_{j} \phi_{j}+\Im\left\{\frac{\beta_{j}}{\phi_{j}} \partial_{t}\left(\overline{\frac{\beta_{j}}{\phi_{j}}}\right)\right\}\right)-\frac{i \partial_{t} d_{j}}{2\left(d_{j}-1\right)} \\
\phi_{j+1} & =\phi_{j}-\frac{2}{1-d_{j}}\left(d_{j} \phi_{j}+\Im\left\{\frac{\beta_{j}}{\phi_{j}} \partial_{t}\left(\overline{\frac{\beta_{j}}{\phi_{j}}}\right)\right\}\right)
\end{aligned}
$$

and

$$
\beta_{j+1}=\frac{1}{1-d_{j}}\left(d_{j} \beta_{j}-i \partial_{t}\left(\frac{\beta_{j}}{\phi_{j}}\right)\right)=-i\left(B_{j+1}\right)_{21}=\overline{-i\left(B_{j+1}\right)_{12}},
$$

where we used the equalities:

$$
\frac{\beta_{j}}{\phi_{j}} \partial_{t}\left(\frac{\overline{\beta_{j}}}{\phi_{j}}\right)=\frac{1}{2} \partial_{t}\left(\left|\frac{\beta_{j}}{\phi_{j}}\right|^{2}\right)+i \Im\left\{\frac{\beta_{j}}{\phi_{j}} \partial_{t}\left(\frac{\overline{\beta_{j}}}{\phi_{j}}\right)\right\}=\frac{\partial_{t} d_{j}}{2}+i \Im\left\{\frac{\beta_{j}}{\phi_{j}} \partial_{t}\left(\frac{\overline{\beta_{j}}}{\phi_{j}}\right)\right\}
$$

and

$$
\frac{\overline{\beta_{j}}}{\phi_{j}} \partial_{t}\left(\frac{\beta_{j}}{\phi_{j}}\right)=\frac{\partial_{t} d_{j}}{2}-i \Im\left\{\frac{\beta_{j}}{\phi_{j}} \partial_{t}\left(\frac{\overline{\beta_{j}}}{\phi_{j}}\right)\right\} .
$$

If $j=1$, then the hypotheses (i) and (ii) are fulfilled. Therefore, our diagonalization procedure works for any $j \leq m-1$ by induction as far as the conditions $d_{j}<1$ hold for $j=1, \ldots, m-1$.

Let us show that the hypothesis (iii) is valid, that is, the estimates $d_{j}<1$ for any $j=1, \ldots, m-1$ are inductively proved. Actually, such estimates can be realized by a suitable choice of the parameter $\varepsilon$ with respect to $\xi$ in the following way:

$$
\varepsilon(\xi)=\min \left\{\tau \in(0, T] ; N=\Theta(\tau)^{\beta}|\xi|\right\}
$$


for a large parameter $N$, to be chosen later.

For non-negative integers $p, q, r$ and a large parameter $N$, we introduce the symbol class $\mathscr{S}_{q}^{p}=\mathscr{S}_{q}^{p}(N, r)$ as follows:

$$
\begin{aligned}
& \mathscr{S}_{q}^{p}(N, r)=\left\{f(t, \xi) \in C^{r}\left((0, T] ; H^{-\infty}\left(\mathrm{R}^{n}\right)\right) ;\right. \\
& \left.\left|\partial_{t}^{k} f(t, \xi)\right| \leq C_{k}|\xi|^{p}\left(N^{-1}|\xi|\right)^{q+k},|\xi| \geq 1,0 \leq k \leq r\right\} .
\end{aligned}
$$

Then we immediately have the following properties:

(i) If $f \in \mathscr{S}_{q}^{p}(N, r)$, then $\partial_{t}^{k} f \in \mathscr{S}_{q+k}^{p}(N, r-k)$.

(ii) If $f_{1} \in \mathscr{S}_{q_{1}}^{p_{1}}\left(N, r_{1}\right)$ and $f_{2} \in \mathscr{S}_{q_{2}}^{p_{2}}\left(N, r_{2}\right)$, then $f_{1} f_{2} \in \mathscr{S}_{q_{1}+q_{2}}^{p_{1}+p_{2}}\left(N, \min \left\{r_{1}, r_{2}\right\}\right)$.

(iii) $\mathscr{S}_{q}^{p}(N, r) \subset \mathscr{S}_{q-k}^{p+k}(N, r)$ and $\mathscr{S}_{q}^{p}(N, r) \subset \mathscr{S}_{q+k}^{p}(N, r)$ for any $k \geq 0$.

These properties imply the following lemma, which ensures the invertibility of $M_{j}=M_{j}(t, \xi)$ for $j=1, \ldots, m-1$.

Lemma 3.3. There exists $N=N(m)$ such that $d_{j} \leq 1 / 2$ for any $1 \leq j \leq$ $m-1$, that is, $\left\{M_{j}(t, \xi)\right\}_{j=1}^{m-1}$ are uniformly invertible in $[0, T] \times \mathbf{R}_{\xi}^{n} \backslash\{\xi ;|\xi|<$ 1\}. Moreover, we have $\lim _{N \rightarrow \infty}\left|M_{j}(t, \xi)-I\right|=0$ for $j=1, \ldots, m-1$, where $|M|=\left(\sum_{k, l}\left|M_{k l}\right|^{2}\right)^{1 / 2}$.

Proof. We note that $\tilde{a}(t, \xi)$ is strictly positive and $\tilde{a}(t, \xi) \in \mathscr{S}_{0}^{0}(N, m)$ by Lemma 3.2, it follows that $\beta_{1} \in \mathscr{S}_{1}^{0}(N, m-1), \tau_{1 \pm}, \phi_{1} \in \mathscr{S}_{0}^{1}(N, m-1)$ and $\left|\phi_{1}\right| \geq C|\xi|$. Moreover, we have $d_{1} \in \mathscr{S}_{1}^{-1}(N, m-1)$, so it follows that there exists $N_{1}>1$ such that $d_{1}<1$ for any $N \geq N_{1}$. Thus $M_{1}$ is invertible for any $N \geq N_{1}$. For $j=1, \ldots, m-2$, let us assume that there exists $N_{j}$ such that $\left\{M_{k}\right\}_{k=1}^{j}$ are invertible for any $N>N_{j}$. If $\beta_{j} \in \mathscr{S}_{j}^{-j+1}(N, m-j)$, $\tau_{j \pm}, \phi_{j} \in \mathscr{S}_{0}^{1}(N, m-j)$ and $\left|\phi_{j}\right| \geq C|\xi|$, then we have $d_{j} \in \mathscr{S}_{1}^{-1}(N, m-$ $j)$. It follows that there exists $N_{j}$ such that $M_{j}$ is invertible. Therefore, by the constructions of $\Phi_{j}$ and $B_{j+1}$, we have $\beta_{j+1} \in \mathscr{S}_{j+1}^{-j}(N, m-j-1)$, $\tau_{(j+1) \pm}, \phi_{j+1} \in \mathscr{S}_{0}^{1}(N, m-j-1)$ and $\left|\phi_{j+1}\right| \geq C|\xi|$ for any $N \geq N_{j}$. Thus the invertibility of $\left\{M_{j}\right\}_{j=1}^{m-1}$ is proved by induction. Here the convergence of $M_{j}(t, \xi ; N)$ to $I$ as $N \rightarrow \infty$ is trivial by $d_{j} \subset \mathscr{S}_{1}^{-1}(N, m-j)$.

Let us choose the parameter $N$ satisfying $d_{j} \leq 1 / 2$ for any $j=1, \ldots, m-1$. We define $\Xi_{m}=\Xi_{m}(t, \xi)$ by

$$
\Xi_{m}(t, \xi)=\left(\begin{array}{cc}
\exp \left(-i \int_{t}^{T} \tau_{m-}(s, \xi) d s\right) & 0 \\
0 & \exp \left(-i \int_{t}^{T} \tau_{m+}(s, \xi) d s\right)
\end{array}\right)
$$


Then we have

$$
\Xi_{m}^{-1}\left(D_{t}-\Phi_{m}+B_{m}+R_{m}\right) \Xi_{m}=D_{t}+\tilde{B}_{m}+\tilde{R}_{m}
$$

where $\tilde{B}_{m}=\Xi_{m}^{-1} B_{m} \Xi_{m}$ and $\tilde{R}_{m}=\Xi_{m}^{-1} R_{m} \Xi_{m}$. Noting the identities

$$
\Re\left\{-i \int_{t}^{T} \tau_{m \pm}(s, \xi) d s\right\}=\frac{1}{2}\left(\log \left(\frac{\tilde{a}(t, \xi)}{\tilde{a}(T, \xi)}\right)+\sum_{j=1}^{m-1} \log \left(\frac{d_{j}(t, \xi)-1}{d_{j}(T, \xi)-1}\right)\right)
$$

we have $\left|\Xi_{m}(t, \xi)\right| \leq 2^{\frac{m-1}{2}} \sqrt{\tilde{a}(t, \xi) / \tilde{a}(T, \xi)}$. Thus we come to the following proposition:

Proposition 3.1. For any small positive constant $\delta$, there exists $N>1$, invertible matrices $\left\{M_{j}\right\}_{j=1}^{m-1}$, diagonal matrix $\Xi_{m}$, and matrices $\tilde{B}_{m}$ and $\tilde{R}_{m}$ satisfying

$$
\begin{gathered}
\sum_{j=1}^{m-1}\left|M_{j}(t, \xi)-I\right| \leq \delta, \quad\left|\Xi_{m}(t, \xi)-\sqrt{\frac{\tilde{a}(t, \xi)}{\tilde{a}(T, \xi)}} I\right| \leq \delta, \\
\left|\tilde{B}_{m}(t, \xi)\right| \leq C|\xi|^{-m+1}\left(\left(\frac{1}{\Theta(t)^{\beta}}\left(\frac{\Theta(t)}{t}\right)^{\frac{1}{m}}\right)^{m} \phi(t ;[\varepsilon(\xi) / 2, T])\right. \\
\left.+\varepsilon^{-m-1} \Theta(\varepsilon(\xi)) \phi(t ;[0, \varepsilon(\xi)])\right)
\end{gathered}
$$

and

$$
\int_{0}^{T}\left|\tilde{R}_{m}(\tau, \xi)\right| d \tau \leq C|\xi| \Theta(\varepsilon(\xi))
$$

such that the following equality holds:

$$
\left(D_{t}+\tilde{B}_{m}+\tilde{R}_{m}\right) \Xi_{m}^{-1} M_{m-1}^{-1} \cdots M_{1}^{-1} M_{0}^{-1} V=0 .
$$

Proof. Let us generalize the symbol class $\mathscr{S}_{q}^{p}$ taking the singularity at $t=0$ into account. For non-negative integers $p, q, r$ and a large parameter $N$, we introduce the symbol class $\mathscr{T}_{q}^{p}=\mathscr{T}_{q}^{p}(N, r)$ as follows:

$$
\begin{aligned}
\mathscr{T}_{q}^{p}(N, r)= & \left\{f(t, \xi) \in \mathscr{S}_{q}^{p}(N, r) ;\left|\partial_{t}^{k} f(t, \xi)\right|\right. \\
& \left.\leq C_{k}|\xi|^{p}\left(\frac{1}{\Theta(t)^{\beta}}\left(\frac{\Theta(t)}{t}\right)^{\frac{1}{m}}\right)^{q+k}, \quad \varepsilon(\xi) \leq t \leq T, \quad 0 \leq k \leq r\right\} .
\end{aligned}
$$


Then $\tilde{a}(t, \xi) \in \mathscr{T}_{0}^{0}(N, m)$, moreover we immediately have the same properties (i)-(iii) on $\mathscr{S}_{q}^{p}(N, r)$. Moreover, we easily see that $\beta_{j}(t, \xi) \in \mathscr{T}_{j}^{-j+1}(N, m-$ $j$ ) for $j=1, \ldots m$ inductively. Thus by Lemma 3.1, Lemma 3.3 and (3.3) we can get all the of properties of Proposition 3.1.

\subsection{Verification of the proof of Theorem 2.1}

Let us conclude the proof of Theorem 2.1. By (2.1) and Proposition 3.1, we have

$$
\begin{aligned}
& \int_{0}^{T}\left|\tilde{B}_{m}(\tau, \xi)\right| d \tau \\
& \leq C|\xi|^{-m+1}\left(\int_{\varepsilon(\xi) / 2}^{T}\left(\frac{1}{\Theta(\tau)^{\beta}}\left(\frac{\Theta(\tau)}{\tau}\right)^{\frac{1}{m}}\right)^{m} d \tau+\varepsilon(\xi)^{-m} \Theta(\varepsilon(\xi))\right) \\
& \leq C|\xi|^{-m+1}\left(\left(\frac{\varepsilon(\xi)}{2}\right)^{m \beta-1} \Theta\left(\frac{\varepsilon(\xi)}{2}\right)^{-m \beta+1} \int_{\varepsilon(\xi) / 2}^{T} \tau^{-m \beta} d \tau+\varepsilon(\xi)^{-m} \Theta(\varepsilon(\xi))\right) \\
& \leq C|\xi|^{-m+1}\left(\Theta(\varepsilon(\xi))^{-m \beta+1}+\Theta(\varepsilon(\xi))^{-m+1}\right) \leq C|\xi|^{-m+1} \Theta(\varepsilon(\xi))^{-m \beta+1} \\
& =C N^{-\frac{m \beta-1}{\beta}}|\xi|^{1-\frac{1}{\beta}} \leq C N^{-\frac{m \beta-1}{\beta}}|\xi|^{\frac{1}{s}}
\end{aligned}
$$

and

$$
\int_{0}^{T}\left|\tilde{R}_{m}(\tau, \xi)\right| d \tau \leq C N^{\frac{1}{\beta}}|\xi|^{1-\frac{1}{\beta}} \leq C N^{\frac{1}{\beta}}|\xi|^{\frac{1}{s}} .
$$

Noting the representation:

$$
W\left(t_{0}, \xi\right)=\left(I+\sum_{k=0}^{\infty} \int_{t_{0}}^{T} \tilde{Q}_{m}\left(t_{1}, \xi\right) \cdots \int_{t_{k}}^{T} \tilde{Q}_{m}\left(t_{k+1}, \xi\right) d t_{k+1} \cdots d t_{1}\right) W(T, \xi),
$$

where $W=\Xi_{m}^{-1} M_{m-1}^{-1} \cdots M_{1}^{-1} M_{0}^{-1} V$ and $\tilde{Q}_{m}(\tau, \xi)=-i\left(\tilde{B}_{m}(\tau, \xi)+\right.$ $\left.\tilde{R}_{m}(\tau, \xi)\right)$, we have

$$
\begin{aligned}
|W(t, \xi)|^{2} & \leq \exp \left(C\left(\int_{0}^{T}\left(\left|\tilde{B}_{m}(\tau, \xi)\right|+\left|\tilde{R}_{m}(\tau, \xi)\right|\right) d \tau\right)\right)|W(T, \xi)|^{2} \\
& \leq \exp \left(C|\xi|^{\frac{1}{s}}\right)|W(T, \xi)|^{2} .
\end{aligned}
$$

Finally, noting $\left|M_{0}^{-1} V(t, \xi)\right|^{2}=2 \tilde{a}(t, \xi)^{2}|\xi|^{2}|v(t, \xi)|^{2}+2\left|v_{t}(t, \xi)\right|^{2}$, and $\tilde{a}_{0} \leq \tilde{a}(t, \xi) \leq \tilde{a}_{1}$ for some positive constants $\tilde{a}_{0}$ and $\tilde{a}_{1}$, we obtain the following uniform estimate in $(0, T] \times \mathrm{R}^{n}$ :

$$
|\xi|^{2}|v(t, \xi)|^{2}+\left|v_{t}(t, \xi)\right|^{2} \leq C \exp \left(C|\xi|^{\frac{1}{s}}\right)\left(|\xi|^{2}\left|\hat{u}_{0}(\xi)\right|^{2}+\left|\hat{u}_{1}(\xi)\right|^{2}\right)
$$


for $\beta>1$, and

$$
|\xi|^{2}|v(t, \xi)|^{2}+\left|v_{t}(t, \xi)\right|^{2} \leq C\left(|\xi|^{2}\left|\hat{u}_{0}(\xi)\right|^{2}+\left|\hat{u}_{1}(\xi)\right|^{2}\right)
$$

for $\beta=1$ respectively; which imply the conclusions of Theorem 2.1 .

\section{Proof of Theorem 2.3}

In this section we prove Theorem 2.3 by an explicit construction of a coefficient $a(t)$. The idea of the proof is based on the method which was developed in the series of papers [1], [2] and [4].

For $v(t, \xi)$ the solution of $(3.1)$, let us define $\mathscr{E}(t, \xi)$ by

$$
\mathscr{E}(t, \xi)=\left|v_{t}(t, \xi)\right|^{2}+a(t)^{2}|\xi|^{2}|v(t, \xi)|^{2} .
$$

Then we have the following property:

Proposition 4.1. For any reference function $\Theta$ satisfying (2.1), for any small positive real number $\kappa$ and for any given positive real numbers $s, \beta$ satisfying $\beta>1$ and $s>\beta /(\beta-1)$, there exist a $(t) \in C^{\infty}((0, T]) \cap L((0, T) ; \Theta)$ satisfying $1 / 2 \leq a(t) \leq 3 / 2$ and (2.3), initial data $\left(\hat{u}_{0}(\xi), \hat{u}_{1}(\xi)\right)$ satisfying

$$
\mathscr{E}(T, \xi) \leq \exp \left(-\kappa|\xi|^{\frac{1}{s}}\right)
$$

for any $\xi \in \mathrm{R}^{n}$, a positive constant $\kappa_{0}$, sequences of positive real numbers $\left\{r_{j}\right\}_{j=1}^{\infty}$ and $\left\{T_{j}\right\}_{j=1}^{\infty}$ satisfying $\lim _{j \rightarrow \infty} r_{j}=\infty$ and $\lim _{j \rightarrow \infty} T_{j}=0$, such that the solutions $v(t, \xi)$ to (3.1) satisfies

$$
\mathscr{E}\left(T_{j}, \xi\right) \geq \exp \left(\kappa_{0}|\xi|^{\frac{\beta-1}{\beta}}\right)
$$

for any $\xi \in\left\{\xi \in \mathbf{R}^{n} ;|\xi|=r_{j}\right\}$ and $j \in \mathbf{N}$.

Proof. Let us take a real, non-negative, $C^{\infty}$ and $2 \pi$-periodic function $\varphi$ such that $\varphi(\tau)=0$ for $\tau$ in a neighborhood of $\tau=0$ and

$$
\int_{0}^{2 \pi} \varphi(\tau)(\cos \tau)^{2} d \tau=\pi
$$

For a positive small constant $\varepsilon$, we define $w(\tau)$ and $\psi(\tau)$ by

$$
w(\tau)=\exp \left(2 \varepsilon \int_{0}^{\tau} \varphi(\sigma)(\cos \sigma)^{2} d \sigma\right) \cos \tau
$$

and

$$
\psi(\tau)=1+4 \varepsilon \varphi(\tau) \sin 2 \tau-2 \varepsilon \nu^{\prime}(\tau)(\cos \tau)^{2}-4 \varepsilon^{2} \varphi(\tau)^{2}(\cos \tau)^{4} .
$$


Here we remark that the any choice of small $\varepsilon$ ensures $1 / 2 \leq \psi(\tau) \leq 3 / 2$. Then $w(\tau), \psi(\tau) \in C^{\infty}(\mathrm{R})$ and satisfy

$$
w^{\prime \prime}(\tau)+\psi(\tau) w(\tau)=0, \quad w(0)=1, \quad w^{\prime}(0)=0 .
$$

For a large natural number $\eta$, to be chosen later, we set the sequences of positive real numbers $\left\{r_{j}\right\}_{j=1}^{\infty}$ and $\{\varrho\}_{j=1}^{\infty}$ by $r_{j}=\eta^{j \beta /(\beta-1)}$ and $\varrho_{j}=2 \pi r_{j}^{-1 / \beta}=$ $2 \pi \eta^{-j /(\beta-1)}$ for $j \in \mathrm{N}$. Moreover, we choose the strictly decreasing sequence of positive real numbers $\left\{t_{j}\right\}_{j=1}^{\infty}$ satisfying $t_{1} / 2 \leq T$,

$$
C_{0}^{-1} \Theta\left(t_{j}\right) \leq \varrho_{j} \leq C_{0} \Theta\left(t_{j}\right) \quad \text { and } \quad \varrho_{j} \Theta\left(t_{j}\right)^{-\beta} \in \mathrm{N}
$$

for a positive constant $C_{0}$. Here we note that $\varrho_{j} \Theta\left(t_{j}\right)^{-\beta} \rightarrow \infty$ as $j \rightarrow \infty$,

$$
\sum_{k=j}^{\infty} \varrho_{k}=\frac{\varrho_{j}}{1-\eta^{-\frac{1}{\beta-1}}} \leq 2 C_{0} \Theta\left(t_{j}\right) \leq \frac{t_{j}}{2}
$$

and

$$
\sum_{k=1}^{j-1} \varrho_{k}^{1-\beta}=\frac{(2 \pi)^{1-\beta}\left(\eta^{j}-\eta\right)}{\eta-1} \leq 2(2 \pi)^{1-\beta} \eta^{j-1}=2 \eta^{-1} \varrho_{j}^{1-\beta}
$$

for $\eta \geq \max \left\{2,2^{\beta-1}\right\}$ and large $j$. We define the coefficient $a(t)$ by

$$
a(t)= \begin{cases}\sqrt{\psi\left(2 \pi \Theta\left(t_{j}\right)^{-\beta}\left(t_{j}-t\right)\right)} & \text { for } t \in\left[t_{j}-\varrho_{j}, t_{j}+\varrho_{j}\right], \\ 1 & \text { for } t \in[0, T] \backslash \bigcup_{j=1}^{\infty}\left[t_{j}-\varrho_{j}, t_{j}+\varrho_{j}\right] .\end{cases}
$$

Then, we see that $a(t) \in C^{\infty}((0, T]) \cap L((0, T) ; \Theta), 1 / 2 \leq a(t) \leq 3 / 2$ and satisfies (2.3) for any $k \in \mathrm{N}$ with small $\varepsilon>0$ from the definition of $\psi$ and (4.4). For a continuous function $v$, to be chosen later, we define $v(t, \xi)$ by

$$
v(t, \xi)=v(\xi) w\left(r_{j}\left(t_{j}-t\right)\right)
$$

for $\xi \in \Omega_{j}:=\left\{\xi ;|\xi|=r_{j}\right\}$ and $j \in \mathrm{N}$. Then $v(t, \xi)$ is a solution to

$$
\left(\partial_{t}^{2}+a(t)^{2}|\xi|^{2}\right) v(t, \xi)=0
$$

with

$$
v\left(t_{j} \pm \varrho_{j}, \xi\right)=v(\xi) \exp \left(\mp 2 \pi \varepsilon|\xi|^{\frac{\beta-1}{\beta}}\right) \quad \text { and } \quad \partial_{t} v\left(t_{j} \pm \varrho_{j}, \xi\right)=0
$$

for $(t, \xi) \in\left[t_{j}-\varrho_{j}, t_{j}+\varrho_{j}\right] \times \Omega_{j}$ and $j \in \mathbf{N}$. Hence we have

$$
\mathscr{E}\left(t_{j} \pm \varrho_{j}, \xi\right)=|\xi|^{2} \nu(\xi)^{2} \exp \left(\mp 4 \pi \varepsilon|\xi|^{\frac{\beta-1}{\beta}}\right),
$$


and it follows that

$$
\mathscr{E}\left(t_{j}+\varrho_{j}, \xi\right)=\mathscr{E}\left(t_{j}-\varrho_{j}, \xi\right) \exp \left(-8 \pi \varepsilon \mid \xi^{\frac{\beta-1}{\beta}}\right)
$$

for $\xi \in \bigcup_{j=1}^{\infty} \Omega_{j}$. Let us prolong $v(t, \xi)$ for all $(t, \xi) \in[0, T] \times \mathrm{R}^{n}$ as the solution of (4.6). Let us note the following estimates:

$$
\begin{aligned}
\int_{t_{j}+\varrho_{j}}^{T}\left|a^{\prime}(\tau)\right| d \tau & =\sum_{k=1}^{j-1} \int_{t_{k}-\varrho_{k}}^{t_{k}+\varrho_{k}}\left|a^{\prime}(\tau)\right| d \tau \leq 2 C_{1} \sum_{k=1}^{j-1} \varrho_{k} \Theta\left(t_{k}-\varrho_{k}\right)^{-\beta} \\
& \leq 2 C_{1} \Theta_{0}^{\beta} \sum_{k=1}^{j-1} \varrho_{k} \Theta\left(t_{k}\right)^{-\beta} \leq 2 C_{0}^{\beta} C_{1} \Theta_{0}^{\beta} \sum_{k=1}^{j-1} \varrho_{k}^{1-\beta} \\
& =4 C_{0}^{\beta} C_{1} \Theta_{0}^{\beta} \eta^{-1} \varrho_{j}^{1-\beta}=4(2 \pi)^{1-\beta} C_{0}^{\beta} C_{1} \Theta_{0}^{\beta} \eta^{-1} r_{j}^{\frac{\beta-1}{\beta}}
\end{aligned}
$$

for any $j \in \mathrm{N}$ by (2.3) and (4.5), where $\Theta_{0}=\sup _{t \in(0, T)}\{\Theta(2 t) / \Theta(t)\}$. Differentiating $\mathscr{E}(t, \xi)$ with respect to $t$ and applying Gronwall' lemma, we have

$$
\begin{aligned}
\mathscr{E}(T, \xi) & \leq \mathscr{E}\left(t_{j}+\varrho_{j}, \xi\right) \exp \left(4 \int_{t_{j}+\varrho_{j}}^{T}\left|a^{\prime}(\tau)\right| d \tau\right) \\
& \leq \mathscr{E}\left(t_{j}-\varrho_{j}, \xi\right) \exp \left(-8 \pi \varepsilon|\xi|^{\frac{\beta-1}{\beta}}+16(2 \pi)^{1-\beta} C_{0}^{\beta} C_{1} \Theta_{0}^{\beta} \eta^{-1}|\xi|^{\frac{\beta-1}{\beta}}\right) \\
& \leq \exp \left(-2 \kappa_{0}|\xi|^{\frac{\beta-1}{\beta}}\right)
\end{aligned}
$$

for any $\eta \geq 8(2 \pi)^{-\beta} C_{0}^{\beta} C_{1} \Theta_{0}^{\beta} \varepsilon^{-1}, \xi \in \Omega_{j}$ and $j \in \mathrm{N}$, where $\kappa_{0}=2 \pi \varepsilon$. Consequently, for any given positive real number $\kappa$, under the choice of the initial data $\left(\hat{u}_{0}(\xi), \hat{u}_{1}(\xi)\right)$ with $v(\xi)$ satisfying $|v(\xi)| \leq 1$ and

$$
\nu(\xi)=|\xi|^{-1} \exp \left(-\frac{1}{2}\left(\kappa|\xi|^{\frac{1}{s}}-2 \kappa_{0} \eta\right)\right)
$$

for $\bigcup_{j=1}^{\infty} \xi \in \Omega_{j}$, we have

$$
\mathscr{E}(T, \xi) \equiv \mathscr{E}\left(t_{1}+\varrho_{1}, \xi\right)=|\xi|^{2} v(\xi)^{2} \exp \left(-2 \kappa_{0} r_{1}^{\frac{\beta-1}{\beta}}\right)=\exp \left(-\kappa|\xi|^{\frac{1}{s}}\right)
$$

It follows that

$$
\mathscr{E}\left(t_{j}-\varrho_{j}, \xi\right) \geq \exp \left(2 \kappa_{0}|\xi|^{\frac{\beta-1}{\beta}}\right) \mathscr{E}(T, \xi) \geq \exp \left(\kappa_{0}|\xi|^{\frac{\beta-1}{\beta}}\right)
$$

for any $j \geq N$ with

$$
N=\min \left\{k \in \mathbf{N} ; \kappa_{0} / \kappa \geq \eta^{-\frac{k}{s}\left(s-\frac{\beta}{\beta-1}\right)}\right\} .
$$


Thus we complete the proof the of Proposition 4.1 by setting $T_{j}=t_{j+N}-\varrho_{j+N}$.

\section{Appendix}

\subsection{Proof of Corollary 1.1}

We set $\Theta(t)=t^{\mu+1}$. Then we have

$$
\Theta(t)^{\beta}\left(t \Theta(t)^{-1}\right)^{\frac{1}{m}}=t^{\beta(\mu+1)-\frac{\mu}{m}} .
$$

Thus the condition (1.5) is equivalent to (2.2) for $\sigma=\beta(\mu+1)-\mu / m$. Noting $\beta=(\sigma+\mu / m) /(\mu+1)$, we have $\beta /(\beta-1)=(\sigma+\mu / m) /(\sigma+\mu / m-\mu-1)$ for $\sigma>\mu+1-\mu / m$ and $\sigma=\mu+1-\mu / m$ if $\beta=1$.

5.2. Some estimates for a $(t)$ from Example 2.1

Let us fix $t>0$ and give a large number $N$ such as $(N+1)^{-p}<t \leq N^{-p}$ and $N \geq N_{0}$. Then we have from the definition of $a(t)$ the followings:

$$
\begin{aligned}
& \int_{0}^{t}|a(\tau)-a(0)| d \tau \\
& \quad=\sum_{j=N_{0}}^{\infty} j^{-r} \int_{0}^{t} \chi\left(j^{q}\left(t-j^{-p}\right)\right) d \tau \\
& =\sum_{j=N+1}^{\infty} j^{-q-r}+N^{-r} \int_{N^{-p}-N^{-q}}^{t} \chi\left(N^{q}\left(t-N^{-p}\right)\right) d \tau\left\{\begin{array}{l}
\leq \sum_{j=N}^{\infty} j^{-q-r} \\
\geq \sum_{j=N+1}^{\infty} j^{-q-r} ;
\end{array}\right.
\end{aligned}
$$

it follows that

$$
\int_{0}^{t}|a(\tau)-a(0)| d \tau \simeq N^{-q-r+1}=\left(N^{-p}\right)^{\frac{q+r-1}{p}} \simeq t^{\frac{q+r-1}{p}}
$$

and

$$
\left|a^{(k)}(t)\right|=N^{-r+k q}\left|\chi^{(k)}\left(N^{q}\left(t-N^{-p}\right)\right)\right| \leq C_{k} t^{-k\left(\frac{q}{p}-\frac{r}{k p}\right)} \leq C_{k} t^{-k\left(\frac{q}{p}-\frac{r}{m p}\right)} .
$$

Let $h \in\left(0, N^{-q}\right)$. By mean value theorem we have

$$
\sup _{0<h \leq N^{-q}}\left\{\frac{|a(t+h)-a(t)|}{h^{\frac{r}{q}}}\right\} \leq N^{-q+r}\left|a^{\prime}(t)\right| \leq C t^{\frac{q-r}{p}}\left|a^{\prime}(t)\right| \leq C_{1} .
$$


On the other hand, for a positive real number $\alpha$ we have

$$
\begin{aligned}
\sup _{\substack{t \in(0, T) \\
\tau \in\left(0, N^{-q}\right)}} & \left\{\frac{\int_{0}^{\tau}|a(t+s)-a(t)| d s}{\tau^{\alpha+1}}\right\} \\
& \geq N^{q(\alpha+1)} \int_{0}^{N^{-q}}\left|a\left(N^{-p}+s\right)-a\left(N^{-p}\right)\right| d s \\
& =N^{q(\alpha+1)-r} \int_{0}^{N^{-q}}\left|\chi\left(N^{q} s\right)-1\right| d s \\
& =N^{q(\alpha+1)-r}\left(N^{-q}-\frac{N^{-q}}{2}\right)=\frac{1}{2} N^{q \alpha-r} .
\end{aligned}
$$

Therefore, $a(t) \in C^{r / q}([0, T])$ and satisfies (1.4) for $\alpha=r / q$, but does not satisfy (1.4) for any $\alpha>r / q$. By applying Theorem 1.5 with $\alpha=r / q$ and $\sigma=(m q-r) /(m p),(1.1)$ is $\gamma^{(s)}$ well-posed for

$$
s<\frac{\sigma}{\sigma(1-\alpha)+\frac{\alpha}{m}-1}=\frac{\frac{m q-r}{m p}}{\frac{m q-r}{m p}\left(1-\frac{r}{q}\right)+\frac{r}{m q}-1}=\frac{q}{q-p-r}=\tilde{s}_{m} .
$$

On the other hand, by applying Corollary 1.1 with $\mu=(q+r-1) / p-1$, (1.1) is $\gamma^{(s)}$ well-posed for

$$
s<\frac{\frac{m q-r}{m p}-\frac{p-q-r+1}{m p}}{\frac{m q-r}{m p}-\frac{p-q-r+1}{m p}-\frac{q+r-1}{p}}=\frac{q(m+1)-p-1}{q-p-1+m(1-r)}=s_{m} .
$$

Here we note that $s_{m}$ is strictly increasing with respect to $m$. Therefore, the estimate $s_{1} / \tilde{s}_{m}=(2 q-p-1) / q>1$ ensures $s_{m}>\tilde{s}_{m}$ for any $m \geq 1$.

\section{REFERENCES}

1. Colombini, F., De Giorgi E., and Spagnolo, S., Sur les équations hyperboliques avec des coefficients qui ne dépendent que du temps, Ann. Scuola Norm. Sup. Pisa Cl. Sci. (4) 6 (1979), 511-559.

2. Colombini, F., Del Santo, D., and Kinoshita, T., Gevrey-well-posed for weakly hyperbolic operators with Hölder-continuous coefficients, Math. Scand. 94 (2004) 267-294.

3. Colombini, F., Del Santo, D., and Kinoshita, T., Well-posedness of the Cauchy problem for a hyperbolic equation with non-Lipschitz coefficients, Ann. Scuola Norm. Sup. Pisa Cl. Sci. (5) 1 (2002), 327-358.

4. Colombini, F., and Spagnolo, S., Some examples of hyperbolic equations without local solvability, Ann. Sci. Ecole Norm. Sup. (4) 22 (1989), 109-125.

5. Hirosawa, F., Loss of regularity for the solutions to hyperbolic equations with non-regular coefficients -an application to Kirchhoff equation-, Math. Methods Appl. Sci. 26 (2003), 783-799. 
6. Hirosawa, F., Global solvability for Kirchhoff equation in special classes of non-analytic functions, J. Differential Equations 230 (2006), 49-70.

7. Yamazaki, T., Unique existence of evolution equations of hyperbolic type with countably many singular of degenerate points, J. Differential Equations 77 (1989), 38-72.

DIPARTIMENTO DI MATEMATICA

UNIVERSITÀ DI BOLOGNA

40127 BOLOGNA

ITALY

E-mail: cicognan@dm.unibo.it
DEPARTMENT OF MATHEMATICAL SCIENCES YAMAGUCHI UNIVERSITY

YAMAGUCHI 753-8512

JAPAN

E-mail: hirosawa@yamaguchi-u.ac.jp 